\title{
Modeling the correlated activity of neural populations: A review
}

\author{
Christophe Gardella, ${ }^{1,2}$ Olivier Marre, ${ }^{2}$ and Thierry Mora ${ }^{1}$ \\ ${ }^{1}$ Laboratoire de physique statistique, \\ CNRS, Sorbonne Université, \\ Université Paris-Diderot, \\ and École normale supérieure (PSL University), \\ 75005 Paris \\ ${ }^{2}$ Institut de la Vision, \\ INSERM, CNRS, \\ and Sorbonne Université, \\ 75012 Paris
}

\begin{abstract}
The principles of neural encoding and computations are inherently collective and usually involve large populations of interacting neurons with highly correlated activities. While theories of neural function have long recognized the importance of collective effects in populations of neurons, only in the past two decades has it become possible to record from many cells simulatenously using advanced experimental techniques with singlespike resolution, and to relate these correlations to function and behaviour. This review focuses on the modeling and inference approaches that have been recently developed to describe the correlated spiking activity of populations of neurons. We cover a variety of models describing correlations between pairs of neurons as well as between larger groups, synchronous or delayed in time, with or without the explicit influence of the stimulus, and including or not latent variables. We discuss the advantages and drawbacks or each method, as well as the computational challenges related to their application to recordings of ever larger populations.
\end{abstract}

\section{CONTENTS}

I. Introduction

II. Collective synchronous activity

A. Stimulus-independent models of activity

1. Coarse models

2. Models of population coupling

3. Models of pairwise correlations

4. Adding population coupling to pairwise models

5. Higher-order interactions

6. Restricted Boltzmann machines

B. Population response to a stimulus

1. Complex noise models

2. Joint stimulus-response ME model

3. Models of correlated noise

III. Temporal correlations

A. Stimulus-independent models

1. Adding time to models of synchronous activity

2. Latent dynamics

B. Stimulus-dependent models

1. Transition models

2. Transition models with latent dynamics

IV. Conclusion and future directions

References

\section{INTRODUCTION}

One of the main goals of neuroscience is to understand how the collective activity of neural networks is influenced by sensory inputs and underlies animal behaviour. Most studies in the past century have studied how single neurons represent information and perform computations. Over the last two decades, tech- nological progress has allowed scientists to record increasingly larger populations of neurons, up to complete organisms (Ahrens et al., 2013; Dupre and Yuste, 2017; Prevedel et al., 2014; Wolf et al., 2015). These recordings have revealed a complex picture where neural networks cannot be solely understood from their individual components.

Anatomically, neurons form highly distributed networks. Cortical pyramidal neurons for example can connect to and receive input from up to thousands of neurons (Shepherd, 2004). This connectivity results in complex dynamics, where some neural ensembles tend to respond synchronously. Even in the absence of sensory input, spontaneous activity in sensory areas can take the form of large synchronous oscillations. Information is now thought to be represented not just by the firing rate of specific cells, but by attractors of neural response trajectories (Hopfield, 1982), or even by trajectories themselves (Mazor and Laurent, 2005). Although neural populations are largely redundant (Puchalla et al., 2005; Warland et al., 1997), some information is synergistic, and cannot be recovered by recording single cells (Franke et al., 2016; Schneidman et al., 2011; Zylberberg et al., 2016).

Whereas isolated neurons are reliable components Mainen and Sejnowski, 1995; Nowak et al., 1997), neural network have intrinsic variability (Deweese and Zador, 2004; Stringer et al., 2016). Part of this variability might result from noise building up along successive computations (Faisal et al., 2008). Variability might also be due to internal network dynamics and top-down modulation (Arieli et al., 1996; 
Nienborg et al., 2012; van Vreeswijk and Sompolinsky, 1996; Vogels et al., 2005). This correlated variability might be more than just noise. Correlated fluctuations of a neuron's input, even independent of the stimulus, can increase the reliability of its response to weak stimuli (Shu et al., 2003), an effect called stochastic resonance (Longtin et al., 1991). Correlation between excitatory and inhibitory inputs can also balance neural activity and increase temporal precision (Baudot et al., 2013; Cafaro and Rieke, 2010; Wehr and Zador, 2003). Correlated activity also facilitates signal transmission across neural networks, even for uncorrelated stimuli (Reyes, 2003). Some works even suggest that stimulus-dependent noise correlations could improve the precision of stimulus encoding (Franke et al., 2016; Zvlberberg et al., 2016). Thus, in order to understand how neurons enco de and process information, it is important to characterize their correlated activity.

Here we review multiple approaches that have been proposed to build explicit probabilistic models of the correlated response of populations of neurons. We focus on models of spike trains rather than of firing rates, as multiple works have shown that spike patterns contain more than just their rate (Baudot et al., 2013; Berry et al., 1997; Kavser et al., 2009; Nemenman et al., 2008; Panzeri et al., 2001; Reich et al., 1997; Reinagel and Reid, 2000; Wehr and Laurent, 1996). Spike trains are also a more faithful description of neurons activity, as organisms sense and act in single trials. Spike train models have been used to study multiple features of the neural code in multiple circuits, such as noise correlations in the auditory cortex (Lvamzin et al., 2015), neural dynamics in primary motor, parietal and ventral premotor cortices (Truccolo et al., 2010), error-correcting stimulus encoding and interactions between neurons in the retina (Ganmor et al., 2011b; Schneidman et al., 2006; Schwartz et al., 2012) and in the primary visual cortex (Ohiorhenuan et al., 2010), .

We focus primarily on models whose parameters can be inferred directly from neural recordings. If a model is too simple, it might not capture some structure in data. But if it is too complex, it will be hard to infer and will require a lot of experimental data. In the models we present, neural responses are influenced by three sources: the spiking history of the recorded population, the stimulus, and latent variables representing unobserved sources such as neighboring neurons that were not recorded. The model chosen for a study depends on the pursued goal. If the aim is to decode a stimulus from the neural activity, the model should take this stimulus into account. The model also depends on the recorded area: strong modulations by internal dynamics, modeled for example by dynamical latent variables, might be more adapted to higher level areas such as the prefrontal cortex than to lower level ones such as the cochlea.

The models we review describe correlations in a popu- lation of $N$ neurons. In order to have a unified framework for all models presented here, we represent spike trains as binned responses. The time is divided in bins of equal length, and the response of neuron $i$ in time bin $t$ is represented by the variable $\sigma_{i t}$. Depending on the model, $\sigma_{i t}$ can either be binary ( 1 if there is at least one spike in the bin, 0 otherwise) or an integer corresponding to the number of spikes in the bin. We refer to the former representation as binary responses, and to the latter as spike count responses. The population responses in time bin $t$ is then represented by a vector of dimension $N$, $\boldsymbol{\sigma}_{t}=\left(\sigma_{1 t}, \ldots, \sigma_{N t}\right)$, where the bold font is used for vectors and matrices. Depending on the goal of the study and on the neural population under consideration, the size of time bins can be very different, typically from 1 ms (Pillow et al., 2008; Truccolo et al., 2010) to longer than a second (Franke et al., 2016). Some of these models, such as the generalized linear model, can also be expressed in continuous time, corresponding to a vanishing bin size in our adopted framework.

\section{COLLECTIVE SYNCHRONOUS ACTIVITY}

Neurons in a population are correlated both in time and across neurons. We begin by presenting models of neural responses in a single time bin, which focus on synchronous activity. Synchronous activity has received a lot of attention (Singer and Gray, 1995). Several models aim at telling which neurons fire synchronously (Cocco et al., 2009; Grün et al., 2002; Shlens et al., 2009; Stevenson et al., 2009).

It should be noted that these models do not assume that temporal correlations are absent, but rather choose to focus of synchronous correlations. However, in some cases where correlations between neurons concentrate on short times that are comparable to the choice of the time bin, these models do in fact provide complete and accurate description of the temporal activity.

The number of possible responses grows exponentially with the population size. Even for populations of a few tens of neurons, the distribution of responses is thus impossible to probe experimentally. Multiple models have been proposed, with different degrees of precision. We start with coarse models, with low numbers of parameters, and progressively increase their complexity. Unless explicitly mentioned, all models of synchronous activity are models of binary responses.

\section{A. Stimulus-independent models of activity}

Stimulus-independent models represent the correlated activity of a neural population with no information about the stimulus. This does not mean that correlations between neurons are not due to stimulus, but only that the 
model does not use that information, just as the brain does not since it has no direct access to it. This can be convenient if no model is available to relate neural responses to stimuli, or if not enough experimental data is available to learn such a model, or if one wants to think about how the nervous system can build representations of neural activity.

\section{Coarse models}

Before presenting specific models, we begin by presenting the framework of Maximum Entropy (ME) models. Many of the models presented below are derived from this framework.

ME models are a powerful tool for modeling distributions from which only limited samples can be observed (Javnes, 1957). Here, we use the ME principle to model the distribution of neural responses $P(\boldsymbol{\sigma})$. The core idea of $\mathrm{ME}$ is to search for the most random distribution that reproduces some chosen descriptive statistics. The model is designed so that these statistics have the same value in the model and in data. One first picks several observables of interest, $\mathcal{O}_{n}(\boldsymbol{\sigma})$, and computes their empirical mean:

$$
\left\langle\mathcal{O}_{n}(\boldsymbol{\sigma})\right\rangle_{\mathrm{emp}}=\frac{1}{T} \sum_{1}^{T} \mathcal{O}_{n}\left(\boldsymbol{\sigma}_{t}\right)
$$

where $T$ is the number of samples. For example, the mean of $\mathcal{O}_{n}(\boldsymbol{\sigma})=\sigma_{i}$ is the mean response of neuron $i$. Then one searches for the model of maximum entropy, $S(P)=-\sum_{\boldsymbol{\sigma}} P(\boldsymbol{\sigma}) \log P(\boldsymbol{\sigma})$, amongst all models that reproduce these statistics.

Given the constraints, the choice of maximum entropy can be interpreted as making as few assumptions as possible to avoid introducing biases. The ME model minimizes the worst-case cost of estimation errors, as measured by the log-loss (Grünwald and Philip Dawid, 2004). For the special case of pairwise models applied to retinal data (see below), it was shown that the ME model outperformed other models picked at random within the same constraints, especially for large networks Ferrari et al. (2017).

Maximum entropy distributions can sometimes be viewed as resulting from "shuffling" experiments, where the patterns of activity are scrambled across times and neurons while keeping the constrained statistics constant Okun et al. (2015); Socolich et al. (2005). The equivalence of this method with maximum entropy has been proven in the special case of pairwise correlations Bialek and Ranganathan (2007), and is likely to be general. While it provides a practical way of generating new activity patterns, unlike ME models this technique cannot evaluate the probability of given patterns, and is unable to extract phenomenological parameters such as coupling constants.
By maximizing the entropy using the technique of Lagrange multipliers to enforce the contraints, one can show that the ME model has the following form:

$$
P(\boldsymbol{\sigma})=\frac{1}{Z} \exp \left(\sum_{n} \theta_{n} \mathcal{O}_{n}(\boldsymbol{\sigma})\right)
$$

where $\theta_{n}$ are the Lagrange multipliers. $Z$ is a normalization constant called partition function, which can be hard to infer, but is only needed for specific applications. For conciseness, in the following we will often omit $Z$ and use instead a proportionality sign $\propto$. Solving for the constraints is equivalent to maximizing the likelihood of the observed data, which is a convex problem. By choosing different observables and comparing the corresponding ME model to empirical responses, one can quantify interactions at different orders (Bohte et al., 2000; (Schneidman et al., 2003), and estimate the mutual information between stimulus and responses (Montemurro et al., 2007; Schneidman et al., 2003).

Importantly, although ME model have a common form (eq. 21), they can be very different in practice, depending on the constrained observables. As we will see, some models can be solved exactly from data, while others require advanced computations to approximate them.

Perhaps the simplest description of the collective population activity is the total number of spiking neurons: $K(\boldsymbol{\sigma})=\sum_{i=1}^{N} \sigma_{i}$. In the literature, $K$ is sometimes termed population rate, although it is more of a population count. Tkačik et al. (2013) proposed to model the distribution of responses with the maximum entropy model reproducing the distribution of the spike count, $P(K)$. This model takes the form:

$$
P(\boldsymbol{\sigma}) \propto \exp [g(K(\boldsymbol{\sigma}))]
$$

where the function $g(K)=\ln P(K)-\ln \left(\begin{array}{l}N \\ K\end{array}\right)$ is learned from data. This model is very easy to learn, even for large populations, using only the histogram of the population count $P(K)$. It is equivalent to the homogeneous neural pool model (Amari et al., 2003), where all neurons are considered identical. It can be shown that it is the only model reproducing the spike count while being invariant to permutations of neurons. Montani et al. (2009) used this model to study higher order correlations (i.e. correlations involving more than two neurons) in somatosensory cortex. They showed that interactions between pairs and triplets of cells were sufficient to account for the distribution of the population count $K$ in a population of 24 cells. It remains unclear how this model could also account for the fine structure of correlations between neurons, as it assumes that all neurons are equivalent.

Another simple ME model is one where the mean activity of each neuron is constrained, $\left\langle\sigma_{i}\right\rangle$. These constraints 
lead to a model of independent neurons:

$$
P(\boldsymbol{\sigma}) \propto \exp \left(\sum_{i} h_{i} \sigma_{i}\right)=\exp \left(\boldsymbol{h}^{\boldsymbol{\top}} \boldsymbol{\sigma}\right)
$$

where the coefficients $h_{i}=\ln \left[\left\langle\sigma_{i}\right\rangle /\left(1-\left\langle\sigma_{i}\right\rangle\right)\right]$ control the mean spiking of each neuron. These coefficients are sometimes called fields, by analogy with magnetic fields in physics. This model is very convenient to learn, to generate data or to compute responses likelihood. On the other hand, it is quite limited, as it cannot account for any correlation. This model is often considered as a default model, setting the baseline for the comparison of other models (Köster et al., 2014; Macke et al., 2011).

\section{Models of population coupling}

These coarse models can be combined to define a more general ME model that can still be solved with relative computational ease: the population coupling model, which reproduces the joint probability between each neuron $i$ and the population count, $P\left(\sigma_{i}, K\right)$ Gardella et al., 2016). This model is motivated by recent work suggesting that some neurons are sensitive to the global population activity rather than to the detailed activity of individual neurons (Okun et al., 2015). This model was also proposed elsewhere, in a slightly different form, where it was termed population tracking model (ODonnell et al., 2017).

The model takes the form:

$$
P(\boldsymbol{\sigma}) \propto \exp \left[\boldsymbol{h}_{K(\boldsymbol{\sigma})}^{\top} \boldsymbol{\sigma}\right]
$$

where the field vector $\boldsymbol{h}_{K}$ depends on the value of the population count $K$. The population activity influences the activity of each cell. Conveniently, this model is tractable in the sense that all predictions, as well as the partition function, can be obtained with arbitrary precision using only polynomial operations that scale with $N^{3}$.

The model can be used to measure how much the population activity shapes the precise structure of pairwise correlations, $\left\langle\sigma_{i} \sigma_{j}\right\rangle-\left\langle\sigma_{i}\right\rangle\left\langle\sigma_{j}\right\rangle$. In the salamander retina (Gardella et al., 2016), the population activity explained $50 \%$ of pairwise correlations, which was similar to what was reported in mouse V1 (Okun et al., 2015). Recent application of the population coupling model to the human and monkey cortices showed that the model explained the collective activity well during sleep, but that detailed interactions between specific pairs of neurons mattered during wakefulness (Nghiem et al., 2018). SImilarly, in mouse V1, population rate predicted better pairwise correlations during synchronized states, and very little during desynchronize states (Okun et al., 2015). Thus, while modeling interactions with the global activity drastically simplifies the structure and inference of the distribution of activities, but it provides only a coarse description of responses which may be inappropriate or incomplete depending on the neural context.

A simpler model that only reproduces the distribution of the population count and its coupling with each neuron, $\left\langle K(\boldsymbol{\sigma}) \sigma_{i}\right\rangle$, has also been proposed (Gardella et al. 2016). Interestingly, for a population of 160 neurons in the salamander retina this simpler model predicted pairwise correlations almost as accurately as the complete coupling model, with 50 times less parameters.

\section{Models of pairwise correlations}

Models based on the total population count and individual activities are easy to infer, but they can only provide a limited description of responses. A finer description of the structure of responses is the correlation between pairs of neurons. Several models have been proposed to describe these pairwise correlations.

The maximum entropy model reproducing the firing rates of all cells, $\left\langle\sigma_{i}\right\rangle$, and the correlation between all pairs of neuron $\left\langle\sigma_{i} \sigma_{j}\right\rangle$ has been used extensively to study correlations in the retina or in multiple areas of the brain (Köster et al., 2014; Meshulam et al., 2017; Schneidman et al., 2006; Tang et al., 2008). This model is often called the Ising model and is equivalent to the "Boltzmann Machine" introduced by Hinton et al. (1984). It takes the form:

$$
\begin{aligned}
P(\boldsymbol{\sigma}) & \propto \exp \left(\sum_{i} h_{i} \sigma_{i}+\sum_{j \neq i} J_{i j} \sigma_{i} \sigma_{j}\right) \\
& \propto \exp \left(\boldsymbol{h}^{\top} \boldsymbol{\sigma}+\boldsymbol{\sigma}^{\top} \boldsymbol{J} \boldsymbol{\sigma}\right)
\end{aligned}
$$

where eq. 7 is written in matrix form. $h$ is a line vector of field coefficients, and $\boldsymbol{J}$ is a symmetric matrix of interaction couplings. The Ising model is very similar in structure to the Hopfield model (Hopfield, 1982), a classical model of memory storage.

The performance of the Ising model at representing the distribution of responses is little affected by pruning weak edges (Ganmor et al., 2011b) (i.e. setting small $J_{i j}$ to 0 ), or by limiting interactions to neighbors only (Shlens et al., 2006, 2009). The Ising model is less accurate when responses have strong correlations, e.g. for neighboring cortical cells (Ohiorhenuan et al., 2010) or at short time scales where refractory periods have strong influence on neuron's dynamics (Bohte et al., 2000).

The Ising model works best on relatively small population, for which its number of parameters remains reasonable. It can be used as a building block for coarse-grained models that couple uniform sub-populations of neurons. In the hierarchical model (Santos et al., 2010), neurons are partitioned into $n$ pools $I_{1}, \ldots, I_{n}$. Pools are formed such that neurons in each pool are as homogeneous as possible, and are each modeled by an Ising 
model. Then, interactions between pools only depend on their population counts $K_{1}, \ldots, K_{Q}$, with $K_{a}=\sum_{i \in I_{a}} \sigma_{i}$ :

$$
P(\boldsymbol{\sigma})=Q\left(K_{1}, \ldots, K_{Q}\right) \prod_{I} P\left(\boldsymbol{\sigma}_{I}\right)
$$

where $\sigma_{I}$ is the sub-vector of neurons in pool $I$. The function $Q$ of interaction is given by a ME model corresponding to various choices of constrained observables, e.g. pairwise joint distribution between the spike counts of two pools. Santos et al. (2010) found that for cortical cultures, the peaks of local field potentials (LFP) activity were better described by the hierarchical model than by the Ising model. Such hierarchical models could also prove useful to model the interactions between neurons from multiple areas.

The Ising model is a powerful theoretical concept, and it can reproduce any pairwise correlations exactly, but it is notoriously hard to infer for populations larger than a few tens of neurons, even with advanced computational techniques (Cocco et al., 2009; Ferrari, 2016). Generating data with this model is also computationally demanding, as it requires complex techniques such as Monte Carlo methods. However, computing responses likelihood is easy, up to the normalization constant. This normalization constant is hard to estimate, but it is not always needed, for example if one only wants to compare the likelihoods of different responses.

An alternative to the Ising model for modeling correlations is the cascaded logistic model. This model is not equivalent to the Ising model but it is close in some cases with sparse connectivity (Park et al., 2013b). This model is build by conditioning the activity of neurons on previous ones using logistic models:

$$
P(\boldsymbol{\sigma})=P\left(\sigma_{1}\right) \prod_{i>1} P\left(\sigma_{i} \mid \sigma_{1} \ldots \sigma_{i-1}\right)
$$

with

$$
P\left(\sigma_{i}=1 \mid \sigma_{1} \ldots \sigma_{i-1}\right)=f\left(h_{i}+\sum_{j<i} w_{i j} \sigma_{j}\right)
$$

where $f(x)=1 /\left(1+e^{-x}\right)$ is the logistic function. The interaction weights $w_{i j}$ are learned from data using maximum likelihood estimation. This model is sensitive to the order of neurons, but algorithms have been developed to find orders that make this model close to the Ising model (Park et al., 2013b). Although inferring this model is easier than the Ising model, it is still not trivial. Unlike the Ising model, it cannot reproduce all correlations exactly, and may even get the mean activities very wrong. The cascaded logistic model is very convenient to generate data and to calculate the likelihood of responses.

Other methods have been proposed to generate responses with fixed pairwise correlations. Both models we present here are common input models, where responses are influenced by a latent input process. Although these models are not guaranteed to exist for any given covariance matrix, such methods are relatively simple theoretically and convenient to generate data.

A first method is the spike train mixture. Several similar techniques have been proposed, which have latter been unified and generalized by Brette (2009). The mixture method was initially introduced for spike trains without response binning. We present it here in the context of binned spike count responses for consistency. One starts by generating $M$ latent Poisson processes, called spike train sources, with rates $\nu_{1}, \ldots, \nu_{M}$. Then, each spike of source $j$ is inserted in the response of neuron $i$ with probability $w_{i j}$. The response of neuron $i$ is then a Poisson process with rate:

$$
\lambda_{i}=\sum_{j} w_{i j} \nu_{j}
$$

and with correlation between neurons $i \neq i^{\prime}$ :

$$
\left\langle\sigma_{i} \sigma_{i^{\prime}}\right\rangle=\sum_{j} w_{i j} w_{i^{\prime} j} \nu_{j}
$$

Brette (2009) presented methods to find appropriate rates $\nu_{1}, \ldots, \nu_{M}$ and mixing matrix $\boldsymbol{w}$ given target firing rates and covariances. Unfortunately, such a solution may be degenerate, and does not always exist for all target correlations; in particular the model cannot produce negative correlations. This model is not equivalent to the Ising model and it introduces additional effective higherorder interactions. The likelihood of responses under the model is not straightforward to compute.

The dichotomized Gaussian model (Amari et al., 2003), another popular common input model for pairwise correlations, is easy to use in practice. Neurons are driven by a Gaussian latent variable $\boldsymbol{z} \sim \mathcal{N}(\boldsymbol{\mu}, \boldsymbol{C})$ of dimension $N$. Neuron $i$ has a binary response: it spikes if $z_{i}$ is positive, else it is silent. A more general model has also been proposed allowing for non-binary spike counts with arbitrary distributions (Macke et al., 2009). Macke et al. (2009) presented efficient ways to find parameters $\boldsymbol{\mu}$ and $\boldsymbol{C}$ agreeing with the data, although such a solution does not always exist, for example in some cases with strongly negative correlations.

Like the spike train mixture model, this model implies higher-order interactions and is distinct from the Ising model, although the importance of this difference is still debated. Macke et al. (2009) showed that for many parameters, the entropy of the dichotomized Gaussian model is close to the entropy of the maximum entropy model, compared to the spike train mixture model. On the other hand, Yu et al. (2011) found that when modeling negative deflations in local field potential (LFP) in the macaque premotor cortex, the dichotomized Gaussian and Ising models made different predictions for response statistics, and the former made predictions closer 
to data than the latter. Recently Lvamzin et al. (2015) used a dichotomized Gaussian model to explain the dependence between stimulus and noise correlations observed in vitro in mice and in vivo in gerbil auditory cortex.

All four models of pairwise correlations - Ising, cascaded logistic, spike train mixture, and dichotomized Gaussian - have about the same number of parameters, but very different computational costs for inference. The most random model, i.e. the Ising model, is surprisingly the hardest to train. When the goal is to reproduce mean activities and pairwise correlations exactly, with the guarantee that no higher-order interactions are present, the Ising model should be preferred. The Ising model can also be used to study the structure of the probability distribution, such as its basins of attraction Loback et al., 2018; Tkacik et al., 2014; Watanabe et al., 2014), or thermodynamic properties (e.g. closeness to a critical point, see Tkacik et al. $(2015))$. On the other hand, if one simply needs to generate responses with a model able to reproduce correlations in most cases, the dichotomized model may be preferred.

\section{Adding population coupling to pairwise models}

It was recently observed that for large populations of over 100 neurons in the salamander retina, the Ising model does not predict interactions between neurons higher than second order (Ganmor et al., 2011a; Tkacik et al., 2014). For example, it fails to estimate the distribution of the population count, over-estimating by more than an order of magnitude the probability of high values of the population count $K$. Several works have attempted to correct this bias.

The most straightforward strategy to correct the Ising model is to add the distribution of the population count to the set of observables constrained by the ME model. The resulting $K$-pairwise model is the maximum entropy model reproducing the mean spiking activities, pairwise correlations, population count histogram $P(K)$ (Tkacik et al., 2014):

$$
P(\boldsymbol{\sigma}) \propto \exp \left[\boldsymbol{h}^{\boldsymbol{\top}} \boldsymbol{\sigma}+\boldsymbol{\sigma}^{\boldsymbol{\top}} \boldsymbol{J} \boldsymbol{\sigma}+g(K(\boldsymbol{\sigma}))\right]
$$

where the function $g$ is learned jointly with $\boldsymbol{h}$ and $\boldsymbol{J}$ from data. Learning this model on the activity of the salamander retina improved the prediction of triplet correlations over the Ising model.

Another model generalizing the Ising model is the semiparametric energy-based probabilistic model where the exponential in eq. 7 is replaced by an arbitrary increasing function $f$ learned from data along with the fields $\boldsymbol{h}$ and couplings $\boldsymbol{J}$ (Humplik and Tkačik, 2016):

$$
P(\boldsymbol{\sigma}) \propto f\left(\boldsymbol{h}^{\top} \boldsymbol{\sigma}+\boldsymbol{\sigma}^{\boldsymbol{\top}} \boldsymbol{J} \boldsymbol{\sigma}\right) .
$$

A non-exponential $f$ can be useful to correct the errors of the Ising model, for example by decreasing the probability of rare events such as high population counts. Such a correction can be applied to any maximum entropy model, and is not specific to the Ising model. Humplik and Tkačik (2016) showed that the semiparametric model indeed described salamander retinal ganglion cell responses better than the Ising model, as measured by data likelihood. This difference increased with the population size. This was achieved even though the semi-parametric model nonlinearity $f$ was parametrized with a low number of parameters.

Unlike the $K$-pairwise model, the semi-parametric model is not guaranteed to reproduce the mean activities and pairwise correlations, and its parameters are less easily interpretable. It should also be noted that the good performance of both models in the retina is not guaranteed to hold in cortical networks, where correlations are stronger.

\section{Higher-order interactions}

Higher-order correlations in neural populations can be hard to model, yet they can have a strong influence on the structure of responses, e.g. for neighboring cortical cells (Ohiorhenuan et al., 2010). The models presented so far have relatively simple interactions, either pairwise interactions, or interactions pooling large numbers of neuron together, regardless of their identity. This restriction is set by the amount of experimental data, which in turn limits the number of parameters. And as we will see now, modeling higher order correlations can require large numbers of parameters.

Martignon et al. (1995) proposed to measure interactions between neurons by writing models in the following form:

$$
P(\boldsymbol{\sigma}) \propto \exp \left(\sum_{i} \theta_{i} \sigma_{i}+\sum_{i_{1} i_{2}} \theta_{i_{1} i_{2}} \sigma_{i_{1}} \sigma_{i_{2}}+\ldots+\theta_{1 \ldots N} \sigma_{1} \ldots \sigma_{N}\right)
$$

This form, called full log-linear model or $\theta$-coordinates system, is general in the case of binary variables: any distribution can be written in this form. The interaction of order $m$ between neurons $i_{1}, \ldots, i_{m}$ is set by the coefficient $\theta_{i_{1} \ldots i_{m}}$, with a value 0 corresponding to no interaction. The ME model reproducing correlations up to maximum order $m$ has all coefficients for higher order than $m$ set to 0 , defining a hierarchy of models as $m$ increases (Schneidman et al., 2003). In this hierarchy, coefficients of order $m$ quantify interaction between neurons that cannot be explained by lower order interactions. For example, the Ising model only reproduces pairwise correlations, so it has no coefficient of order higher than 2. Writing a model in this form can be convenient to estimate interactions between small groups of neurons 
(Yu et al., 2011).

The situation is more complex for large populations. As the $\theta$-form is just a re-writing of response distributions, it has the same number of degrees of freedom, $2^{N}-1$, and it is often impossible to estimate the coefficients empirically. Models have thus been proposed to lower the number of interactions to estimate. A first idea could be to make coefficients $\theta_{i_{1} \ldots i_{m}}$ only depend on the number of neurons, $m$, but not on their identity. This model is invariant to permutations of neurons, and is equivalent to the homogeneous pool model, eq. 3. If permutation invariance is only required for terms of order 3 or more, then one obtains the $K$-pairwise model of eq. 13 .

Another way to limit the number of parameters is to include interaction terms without assuming their order a priori. In the reliable interaction model (Ganmor et al., 2011a), all coefficients are set to 0, except the coefficients necessary to fit the probability of most frequent responses (i.e. the responses $\boldsymbol{\sigma}$ occurring at least $n_{\mathrm{RI}}$ times in recordings). The coefficients are then determined by a system of linear equations matching the model and data values of $\ln P(\boldsymbol{\sigma})$ for these responses. For example, denoting by 0 the state with no spiking neuron, the log-probability that only neuron $i$ spikes is $\theta_{i}+\log P(0)$; the $\log$-probability that only neurons $i$ and $j$ spike is $\theta_{i}+\theta_{j}+\theta_{i j}+\log P(0)$, etc.

This model is convenient because it makes no a priori assumption about the maximum order of interactions, and does not require to learn all low order interactions. For a population of approximately $100 \mathrm{neu}-$ rons in the salamander retina, the reliable interaction model was a more accurate description of frequent responses than the Ising model, with 10 times less parameters (Ganmor et al., 2011a).

This model has several drawbacks. The number of interactions involved in the $n_{\mathrm{RI}}$ most frequent responses is likely to grow exponentially with the population size, which would limit the number of responses used for learning. The coefficients not involved in frequent responses are assumed to be 0 , but this is not constrained by data, which could lead to dramatically over-estimating the probability of unobserved responses, because strongly negative coefficients would be needed to lower the probability of such responses. The model is also not constrained to be normalized. As a result, the model cannot be used to estimate data likelihood, nor to generate patterns of activity, and is limited to a descriptive usage.

\section{Restricted Boltzmann machines}

As we have seen, it is hard to model explicitly general higher-order correlations between neurons. An alternative way to model complex interactions is to introduce latent (i.e. not observed) variables (called hidden units when they are discrete) each interacting with several neurons. Neurons interacting with a common latent variable are effectively correlated through it. Latent variables can account for correlations of different orders. Although latent variables can sometimes be interpreted as originating from an unobserved input, such as common stimuli or unrecorded cells, they do not necessarily correspond to an existing entity. As we will see, latent variable can drastically simplify models.

The restricted Boltzmann machine (RBM) is a simple model of binary neurons interacting with binary hidden units (Smolensky, 1986). Neurons have pairwise interactions with hidden units. While the Ising model is also a Boltzmann machine, this model is said to be restricted, as not all pairwise interactions are allowed: there is no direct interaction between two neurons or between two hidden units. The joint probability between neurons and hidden units is thus:

$$
P(\boldsymbol{\sigma}, \boldsymbol{z}) \propto \exp \left(\boldsymbol{a}^{\boldsymbol{\top}} \boldsymbol{\sigma}+\boldsymbol{b}^{\boldsymbol{\top}} \boldsymbol{z}+\boldsymbol{z}^{\boldsymbol{\top}} \boldsymbol{w} \boldsymbol{\sigma}\right)
$$

where $\boldsymbol{z}$ is the column vector of binary hidden units of size $M$, the number of hidden units. Fields $\boldsymbol{a}$ and $\boldsymbol{b}$ are vectors controlling the activity of neurons and hidden units, and $\boldsymbol{w}$ is a matrix of interaction coupllings of size $M \times N$. $\boldsymbol{a}, \boldsymbol{b}$ and $\boldsymbol{w}$ are learned from data by likelihood maximization, which can be done relatively easily using algorithms such as contrastive divergence (Hinton, 2002) or persistent contrastive divergence (Tieleman, 2008). The probability of responses is obtained by marginalizing over latent variables, which can be computed analytically, up to a normalization constant (Fischer and Igel, 2012).

The number of hidden units is left to choice, and can be larger than the number of neurons. A compromise must usually be made: the more hidden units, the more complex distributions can be represented, and the more data is needed for inference. Any binary distribution can be approximated with arbitrary precision by an RBM, as measured by the Kullback-Leibler divergence (Le Roux and Bengio, 2008), illustrating the fact that arbitrary complex correlations between neurons can be described by simple interactions with latent variables. A very large number of hidden units (and parameters) might be required to achieve arbitrary precision. Because of the risk of overfitting, the RBM is not guaranteed to be an accurate model for experimental recordings of limited duration. Also, the RBM does not allow for an easy interpretation in terms of interactions between neurons, as the $\theta$-form does (eq. 15).

The restricted nature of the RBM is convenient for computations: given a state of latent variables, neurons are independent, and conversely. This make the RBM very convenient to learn and to generate data. The RBM has been shown to be a more accurate model than the Ising model for responses in the cat visual cortex (Köster et al., 2014). Humplik and Tkačik (2016) also 
found that in the salamander retina, the RBM gave a better description of responses compared to the $K$-pairwise and semi-parametric energy-based models, in terms of response likelihood. Similar observations were reported in the rat retina (Gardella et al., 2018). This difference was accentuated for larger population sizes.

The RBM can be enhanced by also allowing interactions between neurons to define a semi-restricted Boltzmann machine (Köster et al., 2014). For populations in the cat primary visual cortex of up to 36 cells, little difference was found between the RBM and the semi-RBM. In this case, the RBM is not only a better model of neural responses than the Ising model, but it is also as efficient as the Ising model at capturing pairwise correlations. As the size of the population considered was rather limited, it would be interesting to test if such results also hold for larger populations.

The RBM is thus a very promising model, which outperforms almost all models of synchronous population activity presented so far. Its main drawback is the lack of interpretability of its parameters. It would be interesting to ask whether the performance of the RBM extends to modeling the activity of large populations of cortical neurons in behaving animals.

\section{B. Population response to a stimulus}

A significant part of correlations between sensory neurons can be due to the stimulus. Different neurons may respond to common features of the stimulus, and be correlated through this shared influence. Stimulus features may themselves be correlated with other, inducing more correlations downstream. Including the influence of the stimulus on neural responses should provide a better description of correlated responses. On the other hand, even for a given stimulus, correlations between the noisy responses may remain. Taking those noise correlations into account can significantly improve the description of responses to stimuli, and thus improve decoding precision (Franke et al., 2016; Zylberberg et al., 2016). An extensive part of the neuroscience literature has focused on modeling the influence of a stimulus $s$ on responses, $P(\boldsymbol{\sigma} \mid \boldsymbol{s})$. A complete review of such models is beyond the scope of this review. Here, we focus on models of response where correlations cannot be explained by the stimulus alone, or in other words where noise correlations are present.

The framework presented here can describe both correlations with a stimulus or with behaviors Lawhern et al., 2010). Behaviors can correlate both with motor neurons controlling them, but are also known to modulate sensory neurons (Nienborg et al., 2012). In general, the models presented here correlate neural responses to an external variable $s$, without assuming the direction of causality. We shall refer to $s$ as "stimulus" for simplicity, but it can also be understood as behavior, such as arm movements.

\section{Complex noise models}

A natural strategy is to model the probability of responses to each stimulus $s, P(\boldsymbol{\sigma} \mid s)$, using any model previously described. This method can model complex noise correlations between neurons. If the set of stimuli is discrete, then the amount of parameters to estimate would grow linearly with the number of stimuli. In order to limit the amount of parameters to estimate, one can impose constraints on the parameters, e.g. that some parameters are constant across stimuli. In the stimulus-dependent Ising model, each distribution $P(\boldsymbol{\sigma} \mid \boldsymbol{s})$ is modeled by an Ising model (eq. 7). Schaub and Schultz (2012) applied this model to a population of orientation selective neurons, and showed how decoding could be achieved in this framework, using simulations. But this direct approach used 10000 responses per stimulus, which is challenging experimentally.

Another possible approximation is to consider that in eq. 7 fields $\boldsymbol{h}$ depend on the stimulus, but not the couplings $\boldsymbol{J}$. A challenge is then to model how fields $\boldsymbol{h}$ depend on stimulus $\boldsymbol{s}$. Granot-Atedgi et al. (2013) modeled it with a Linear Nonlinear model:

$$
h_{i}(s)=f_{i}\left(\boldsymbol{k}_{i}^{\top} \boldsymbol{s}\right)
$$

where $\boldsymbol{k}_{i}$ is a linear filter spanning the stimulus space, and $f_{i}$ is a nonlinear function. The success of this strategy was made possible by the simplicity of the stimulus and neurons used: retinal ganglion cells were stimulated by a uniform time-varying light intensity. But often, there is no simple model to predict how even single neurons respond to a stimulus, e.g. natural movies (Gollisch and Meister, 2010; McIntosh et al., 2016).

A helpful trick is to use time-dependent models instead of stimulus-dependent ones (Ganmor et al., 2015; Granot-Atedgi et al., 2013; Köster et al., 2014; Tkačik et al., 2010). One records multiple responses to repetitions of a stimulus, and computes the mean response for each time bin, $\left\langle\sigma_{i t}\right\rangle$, also called peristimulus time histogram (PSTH). Then one studies the ME model that reproduces each $\left\langle\sigma_{i t}\right\rangle$, as well as correlations between neurons, $\sum_{t}\left\langle s_{i t} s_{j t}\right\rangle$ :

$$
P\left(\boldsymbol{\sigma}_{t}\right) \propto \exp \left(\boldsymbol{h}_{t}^{\top} \boldsymbol{\sigma}+\boldsymbol{\sigma}^{\boldsymbol{\top}} \boldsymbol{J} \boldsymbol{\sigma}\right)
$$

where fields $\boldsymbol{h}_{t}$ are different for each time bin, and are learned from data along with $\boldsymbol{J}$. This formulation is convenient, as it makes it possible to study interactions between neurons for any stimulus, even if no model is available to predict responses to stimuli in general. Of course, such a model is limited as it requires stimulus repetitions and it cannot make predictions for responses to new stimuli. Nevertheless, a convenient strategy to generalize this 
model is to first learn time-dependent fields $\boldsymbol{h}_{t}$ and couplings $\boldsymbol{J}$, and combine it with a single-neural model of encoding predicting $\left\langle\sigma_{i t}\right\rangle$ to calculate a stimulus-dependent $h_{i t}=h_{i}(\boldsymbol{s})$. This avoids optimizing $\boldsymbol{J}$ and $\boldsymbol{h}(\boldsymbol{s})$ at the same time, which is computationally challenging and may also wrongly attribute to noise correlations some signal correlations that are missed by the single-neuron model. Ferrari et al. (2018) applied this strategy to populations of retinal ganglion cells responding to moving bars, and showed that the inferred couplings $\boldsymbol{J}$ generalized very well to other stimulus ensembles.

Köster et al. (2014) proposed to add stimulus dependence to both restricted and semi-restricted Boltzmann machines (eq. 16). In order to keep the number of stimulus-dependent parameters low, they considered a model where neuron the fields $\boldsymbol{a}(\boldsymbol{s})$ depend on the stimulus, but the couplings $\boldsymbol{w}$ with hidden units and hidden unit fields $\boldsymbol{b}$ do not. As above, a time-dependent $\boldsymbol{a}_{t}$ can be used instead of a stimulus-dependent $\boldsymbol{a}(\boldsymbol{s})$ if stimulus repetitions are available.

\section{Joint stimulus-response ME model}

Another approach to capture the dependence between neurons and stimulus is to model the joint probability of stimuli $s$ and responses $\sigma$ with an ME model. Gerwinn et al. (2009) for example proposed the joint pairwise maximum entropy model, an ME model with pairwise interactions between a continuous stimulus $s$ and binary responses $\boldsymbol{\sigma}$. Similarly to the Ising and RBM models, this model has the form:

$$
P(\boldsymbol{s}, \boldsymbol{\sigma}) \propto \exp \left(\boldsymbol{h}_{1} \boldsymbol{\sigma}+\boldsymbol{\sigma}^{\boldsymbol{\top}} \boldsymbol{J}_{1} \boldsymbol{\sigma}+\boldsymbol{h}_{2} \boldsymbol{s}+\boldsymbol{s}^{\boldsymbol{\top}} \boldsymbol{J}_{2} \boldsymbol{s}+\boldsymbol{s}^{\boldsymbol{\top}} \boldsymbol{J}_{3} \boldsymbol{\sigma}\right)
$$

where vectors $\boldsymbol{h}_{1}$ and $\boldsymbol{h}_{2}$ control the mean of $\boldsymbol{\sigma}$ and $\boldsymbol{s}$, and matrices $\boldsymbol{J}_{1}, \boldsymbol{J}_{2}$ and $\boldsymbol{J}_{3}$ control the correlation between them. They are all learned jointly from data. Conditioned by a response, the stimulus has a Gaussian distribution, and conditioned by a stimulus, responses have an Ising model distribution. The structure of the model puts hard constraints on the statistics of the stimulus. Once marginalized over responses, the stimulus distribution is a mixture of Gaussians, which might be problematic for example if the stimulus used in an experiment is not well approximated by one.

A variant is partially dichotomized Gaussian model (Cox and Wermuth, 1999; Gerwinn et al., 2009), which combines the ideas of the dichotomized Gaussian model and of the joint pairwise ME model. A latent variable $\boldsymbol{z}$ is considered, such that the joint distribution between the stimulus $s$ and the latent variable $z$ is Gaussian. Then, neuron $i$ spikes if latent variable $z_{i}$ is positive. The distribution of stimuli marginalized over responses is also Gaussian, making it only suitable for specific experiment designs.
These models have convenient properties for analysis. For example one can easily compute the spike-triggered average of each neuron or group of neuron. However, it is hard to interpret directly the parameters of these models.

\section{Models of correlated noise}

We have presented models of stimulus-dependent neural populations where the noise could be relatively complex. But it has been suggested that the noise in some populations might have a simple form, typically a global modulation of firing rates (Schölvinck et al., 2015). Some models have been proposed to capture this simple kind of noise. They are easier to use than previous models, as they tend to have simple structures. In the following, we assume that neurons' firing rates are influenced by stimulus $s$ through a deterministic sensory drive, $\phi(s)$, characterized by the neurons' tuning curves. Given a firing rate, neuron responses are noisy, as typically captured by a Bernoulli (for binary variable) or a Poisson (for spike counts) process. Here we assume that the firing rate $\boldsymbol{\lambda}$ is also a stochastic process, correlated across neurons. Such models are called doubly stochastic Poisson, or Cox processes.

Arieli et al. (1996) suggested that the activity of cortical sensory neurons could be described by summing a stimulus drive to the spontaneous activity. Schölvinck et al. (2015) showed that the variability of cat V1 neurons was mostly shared. Furthermore, an additive shared variability could partially account for the dependence of pairwise correlations on neural states. The rectified Gaussian model (Bányai et al. (2016), derived from Carandini (2004)) captures this shared variability. Correlation between neurons is represented by a latent correlated variable $\boldsymbol{z}$ of dimension $N$, independent of the stimulus. The firing rate of neuron $i$ is:

$$
\lambda_{i}=f_{r}\left(\phi_{i}(s)+z_{i}\right)
$$

where the rate function $f_{r}(x)=k\left[x-V_{0}\right]_{+}^{\beta}$ is a rectifying function. The latent variable $\boldsymbol{z}$ is a centered Gaussian variable with covariance learned from data. Importantly, $\boldsymbol{z}$ is independent from the stimulus. Yet, since the rate function $f_{r}$ is non-linear, the amplitude of the noise does depend on the stimulus. Bányai et al. (2016) applied this model to population of neurons in macaque V1, and found that it could account for part of the response variability.

Other works showed that the shared variability is not limited to additive interactions, but also takes the form of common multiplicative changes (Ecker et al., 2014), possibly in the form of transient 50-100 ms packets of spiking activity (Luczak et al., 2013). In the affine population modulation model (Lin et al., 2015), the firing rates are correlated both in gain and in offset:

$$
\lambda_{i}=z_{i}^{(1)} \phi_{i}(\boldsymbol{s})+z_{i}^{(2)}
$$


where $\boldsymbol{z}^{(1)}$ and $\boldsymbol{z}^{(2)}$ are latent variables with correlations across neurons. $\boldsymbol{z}^{(1)}$ has mean 1 , whereas $\boldsymbol{z}^{(2)}$ has mean 0 . In the extreme case where $\boldsymbol{z}^{(1)}$ is always 1 the noise is purely additive, whereas if $\boldsymbol{z}^{(2)}$ is always 0 the noise is purely multiplicative. If $\boldsymbol{z}^{(1)}$ is always 1 and $\boldsymbol{z}^{(2)}$ always 0 , there is no shared variability. Lin et al. (2015) found that in cat V1, both additive and multiplicative common noise where involved, with relative importance varying in time.

Franke et al. (2016) considered a similar, although slightly more complex model for correlations between cells:

$$
\lambda_{i}=z_{i}^{(1)} \times f_{i}\left(z_{i}^{(2)} \phi(s)+z_{i}^{(3)}\right) .
$$

$\boldsymbol{z}^{(1)}, \boldsymbol{z}^{(2)}$ and $\boldsymbol{z}^{(3)}$ are 3 vectors of correlated latent variables independent of the stimulus and of each other, with $\left\langle\boldsymbol{z}^{(1)}\right\rangle=\mathbf{1},\left\langle\boldsymbol{z}^{(2)}\right\rangle=\mathbf{1}$, and $\left\langle\boldsymbol{z}^{(3)}\right\rangle=\mathbf{0}$. As in the rectified Gaussian model, when $f_{i}$ is nonlinear the amplitude of the noise due to $\boldsymbol{z}^{(2)}$ and $\boldsymbol{z}^{(3)}$ depends on the stimulus, which is not the case for $\boldsymbol{z}^{(1)}$. The parameters of the model can be fit using a multi-step procedure based on a series of mean squared difference minimizations. Interestingly, Franke et al. (2016) showed that for direction selective cells in the rabbit retina, the stimulus-dependent noise captured by $\boldsymbol{z}^{(2)}$ was beneficial to stimulus encoding.

At this point it should be emphasized that the latent variables $\boldsymbol{z}$ discussed in this section have a very different interpretation than for stimulus-independent model, where those variables accounted for stimulus-induced correlations. Here, latent variable only reflect noise correlations, even though their impact may depend on the stimulus itself because of nonlinearities. Even for a fixed stimulus, latent variables can be used to account for two different sources of variability. The first one, $\boldsymbol{z}$, which we have discussed so far, is the variability across the population likely to occur in real life conditions, due to neural network dynamics, such that repetitions of a stimulus evoke different responses even if repetitions are close in time. The other source of variability, denoted by $\boldsymbol{\zeta}$, represents fluctuations in activity on the time scale of experiments (e.g. fluctuations in neurons firing rate), which are likely due to experimental conditions. Often, there is no clear distinction between $\boldsymbol{z}$ and $\boldsymbol{\zeta}$, although $\boldsymbol{\zeta}$ is constrained to have slow variations. In some models $\boldsymbol{z}$ and $\boldsymbol{\zeta}$ are summed to represent variability on short and long time scales respectively (Park et al., 2015). The modulated Poisson model is an example where the firing rate is modulated by the latent variable $\zeta$ (Rabinowitz et al., 2015):

$$
\lambda_{i}=\zeta_{i} \times \phi_{i}(s)
$$

This model is in principle equivalent to eq. 22 with $\boldsymbol{z}^{(2)}=\mathbf{1} \boldsymbol{z}^{(3)}=\mathbf{0}$, but with the additional constraint that $\zeta_{t}$ varies slowly in time to reflect long-term fluctuations of the cell states. In practice, this condition is enforced by setting $\log \zeta_{t}$ to be a Gaussian process with strong temporal correlations. Rabinowitz et al. (2015) applied this model to extracellular recordings of single auditory neurons in the primary auditory cortex and midbrain of anesthetized ferrets. They showed that the modulated Poisson model can be used to infer the influence of the stimulus on responses with a higher precision than a model without modulation.

\section{TEMPORAL CORRELATIONS}

So far we have described models of responses of $N$ neurons in a single time bin. Neural populations also have strong temporal correlations of their activity across different time bins, and a variety of models have been proposed to describe these correlation by spanning multiple time bins.

\section{A. Stimulus-independent models}

\section{Adding time to models of synchronous activity}

A natural way to generalize models for single time bins to responses spanning $B$ time bins is to consider that each neuron in each time bin as a different neuron. We obtain a population of $N B$ activity units, to which we can apply any model of activity proposed for a single time bin. This process is sometimes called time spatialization (Buonomano and Maass, 2009). There are several examples of this strategy, such as the spatio-temporal Ising model (Ganmor et al., 2011a; Köster et al., 2014; Marre et al., 2009), the spatio-temporal reliable interaction model (Ganmor et al., 2011a) or the spatio-temporal restricted Boltzmann machine and semi-restricted Boltzmann machine (Köster et al., 2014). This approach has a major drawback: it cannot be used to describe stationary activity (invariant to time translation). It cannot be used to generate longer spike trains than $B$, or to evaluate their likelihood.

Other models for spatio-temporal interactions are specifically designed for stationary distributions. Vasquez et al. (2012) presented the general form of ME models with temporal interactions for stationary distributions:

$$
P\left(\left\{\boldsymbol{\sigma}_{t}\right\}\right) \propto \exp \left[\sum_{t}\left(\boldsymbol{h}^{\boldsymbol{\top}} \boldsymbol{\sigma}_{t}+\sum_{\tau=1}^{u} \boldsymbol{\sigma}_{t}^{\boldsymbol{\top}} \boldsymbol{J}_{\tau} \boldsymbol{\sigma}_{t+\tau}\right)\right] .
$$

The sum over time in the exponential ensures that this distribution is invariant to time translations, and is a general feature of ME models for stationary distributions. Constraining ME models to account for temporal stationarity reduces the number of parameters to learn 
compared to time spatialization. All time bins share the same field $\boldsymbol{h}$ and the coupling weight $\boldsymbol{J}$ only depend on the time delay $\tau$. The model was applied to the salamander retina using brute-force gradient descent. It was shown that interactions over a $30 \mathrm{~ms}$ delay could predict well cross-correlations in the activity with a longer delay, up to $120 \mathrm{~ms}$. Although Nasser et al. (2013) described Monte Carlo methods to infer the parameters of these models, this inference procedure is computationally very intensive and has only been applied to small populations of neurons.

A simpler alternative is to focus on the evolution of the population count $K_{t}=K\left(\sigma_{t}\right)$ instead of tracking individual neurons. Mora et al. (2015) proposed a ME model reproducing the temporal correlations of the population count over a finite delay, $P\left(K_{t}, K_{t+\tau}\right)$ for $\tau=1, . ., u$ :

$$
P\left(\left\{\boldsymbol{\sigma}_{t}\right\}\right) \propto \exp \left(\sum_{t}\left[h\left(K_{t}\right)+\sum_{\tau=1}^{u} J_{\tau}\left(K_{t}, K_{t+\tau}\right)\right]\right),
$$

where the function $\boldsymbol{h}$ controls the distribution of the population count, and the function $\boldsymbol{J}_{\tau}$ controls its temporal correlation at a delay $\tau$. Functions $\boldsymbol{h}$ and $\boldsymbol{J}$ are learned jointly from data. The model was used to show that for a population of 185 neurons in the rat retina, a memory span of only $u=4$ time bins corresponding to a 40 ms delay described well the dynamics of the population count, and in particular the durations and sizes of neural avalanches. This model can be further simplified by considering the spike count as a continuous variable, and constrain only its mean and covariance. The corresponding ME model is then equivalent to a Gaussian process in the spike count $K_{t}$, which can be described by a Gaussian autoregressive process with memory $u$ (Mora et al., 2015). This simplification makes the model very easy to learn, but results in a poor description of the spike count distribution.

Restricted Boltzmann machines can also be adapted to stationary distributions. The temporal restricted Boltzmann machine (Gardella et al., 2018), is a model with hidden units in each time bin, witch can interact with neurons in different time bins:

$P\left(\left\{\boldsymbol{\sigma}_{t}, \boldsymbol{z}_{t}\right\}\right) \propto \exp \left[\sum_{t}\left(\boldsymbol{a}^{\top} \boldsymbol{\sigma}_{t}+\boldsymbol{b}^{\top} \boldsymbol{z}_{t}+\sum_{\tau=1}^{u} \boldsymbol{z}_{t}^{\top} \boldsymbol{w}_{\tau} \boldsymbol{\sigma}_{t+\tau}\right)\right]$

It is a convolutional RBM, so interactions between neurons and hidden units only depend on the delay $\tau$ between them, not on their absolute time. Importantly, once the model parameters are learned, they can be used to model responses of any time length. Gardella et al. (2018) found that this model was an accurate description of temporal correlations for a population of neurons in the rat retina. Furthermore, they showed that much information about the stimulus could be easily read out from the activity of hidden units.
Finally, it should be noted that the common-input models presented earlier for the synchronous activity can also be adapted to model stationary temporal correlations. Brette (2009) presented spike train mixture methods to generate responses with fixed autocorrelations and cross-correlations between neurons, even in the case of non-binned spike trains. The dichotomized Gaussian model can also be generalized to account for temporal correlations (Macke et al., 2009).

\section{Latent dynamics}

In the temporal restricted Boltzmann machine, temporal correlations arise from interactions between neurons and hidden units with a time delay. In a different approach, neural activities only depend on the current value of the latent variables, but these latent variables are correlated in time. Temporal correlations between neurons then arise from temporal correlations in the dynamics of the latent variables. Models built on this idea are usually hard to learn, although expectationmaximization techniques have been proposed for this purpose (Dempster et al., 1977; Yu et al., 2009). This approach can be used to extract low-dimensional dynamics underlying neural responses, sometimes called neural trajectories (Gao et al., 2016). These trajectories are convenient for visualizing data and for decoding responses $\mathrm{Yu}$ et al. (2009). They can also be used to decode movements from neural populations in motor areas Gao et al. (2016); Yu et al. (2009). As these models try to identify potentially simple latent dynamics underlying noisy responses, they are closely related to questions of dimensionality reduction (see Cunningham and Yu (2014) for a review). Much like the restricted Boltzmann machine, latent variables should be viewed as a convenient abstract tool rather than reflecting actual biological processes. Latent variables can be continuous, typically with autoregressive Gaussian dynamics, or categorical, typically with Markov chain dynamics.

In the linear dynamical system, the dynamics of the vector of latent variables $\boldsymbol{z}$ follows a Markovian Gaussian process:

$$
\boldsymbol{z}_{t}=\boldsymbol{A} \boldsymbol{z}_{t-1}+\boldsymbol{\epsilon}_{t}
$$

where $\boldsymbol{A}$ is the dynamics matrix and $\boldsymbol{\epsilon}$ is the innovation noise, typically Gaussian. The neural trajectory $\boldsymbol{z}_{t}$ drives the firing rate of neuron $i$ through a rate function $f_{i}$ :

$$
\lambda_{i t}=f_{i}\left(\boldsymbol{z}_{t}\right)
$$

Stochastic responses are then produced by a Bernoulli, Poisson, or generalized count process. The dimensionality of the latent space is left to choice, and represents a trade-off between model accuracy and data required for 
learning. Gao et al. (2016) proposed a simple exponential nonlinearity $f_{i}$ :

$$
\lambda_{i t}=\exp \left(\mu_{i}+\boldsymbol{C}_{i}^{\boldsymbol{\top}} \boldsymbol{z}_{t}\right)
$$

where $\boldsymbol{C}_{i}$ is a projection vector and $\mu_{i}$ is a constant influencing the neuron's firing rate. Both $f+i$ and $\boldsymbol{C}_{i}$ are learned from data. More complex rate functions $f_{i}$ can be used for more accurate models. In the linear dynamical system with nonlinear observation, $f_{i}$ is adapted to each neuron and can be highly nonlinear. In Gao et al. (2016), this is achieved by learning each $f_{i}$ with a feed-forward neural network model. Gao et al. (2016) shows that the resulting model is able to give a precise description of response dynamics in macaque V1. Furthermore, they are able to precisely correlate macaque hand-reaching directions to neural trajectories from the motor cortex. Yu et al. (2009) used a similar model. They used the same firing rate vector $\boldsymbol{\lambda}_{\boldsymbol{t}}$ driven by a latent variable (eq. 29), but also allowed for interactions between neural responses in the same time bin. They did so by assuming that the square-root of the spike counts approximately followed a multivariate Gaussian distribution with mean $\boldsymbol{\lambda}_{t}$ and covariance $\boldsymbol{R}$ learned from data. The model was used to identify neural trajectories in macaque primary and premotor cortices during motion planning.

Unlike the latent dynamical system, hidden Markov models use latent variables with discrete states (Abeles et al., 1995), which are sometimes called "modes." The stochastic dynamics of the latent variable $\boldsymbol{z}$ is typically characterized by a Markov chain, where the transition matrix is learned from data. The number of states can be high: for a population of 150 cells from a salamander retina, Prentice et al. (2016) reported that the model performance at describing data improved for up to 70 modes. For each latent state $\boldsymbol{z}$, responses are distributed according to an emission distribution $P(\boldsymbol{\sigma} \mid \boldsymbol{z})$. Because one such distribution must be learned for each mode, the size of empirical data usually forbids emission distributions with many parameters. For instance, Prentice et al. (2016) model emission distributions with independent neurons (with mode-dependent mean activities), or with Chow-Liu trees. A Chow-Liu tree is a simplification of the cascaded logistic model (eq. 10), where the response of neuron $i$ only depends on just a single neuron $j<i$ previously characterized in the cascade. As the order of neuron indices is important for this model, it could be different for each latent mode. A Chow-Liu tree can only produce weak correlations for large populations of neurons, but it has a low number of parameters compared to the Ising and cascaded logistic models. In the recordings from the salamander retina, interactions added by the Chow-Liu tree structure resulted in improved predictions for correlations between pairs and triplets of neurons. The hidden Markov model resulted in a better description of responses than the reliable- interaction and $K$-pairwise models, as measured by the likelihood. However, it is unclear how these models could generalize to larger populations, as all neurons from the population share the same latent mode, and the diversity of modes is expected to grow exponentially with the number of neurons, even when correlations are weak.

\section{B. Stimulus-dependent models}

\section{Transition models}

Neural networks are influenced by both stimulus and internal dynamics. We have presented models that account for each effect separately. We now present models where neurons are influenced by both. These models take the form of transition probabilities, which describe the response probability in a single time bin, given the population spiking history in previous time bins and the stimulus. Neurons are often assumed to be independent given this history, and we can then model each neuron independently. We refer to such models as transition models. The two kinds are the generalized linear model and the leaky integrate and fire neuron model. All models presented here share a common structure: inputs drive a current $I$ entering the cell, and this current induces spikes. The input current is described by a linear sum of inputs. A stochastic process describes how the current induces spikes. Models presented here should be considered as abstract descriptions of correlations between inputs and neural activities, rather than an actual causal model describing biological mechanisms driving neural responses. We first start by describing how the input current is influenced by the stimulus and the past activity of the population. Then we will present the different spike generation processes.

All correlations captured by the transition models originate from the inputs driving the current $I$. Here we describe the most common form, where the input current of neuron $i$ in time bin $t$ is influenced additively by the stimulus and by the population activity in previous time bins $\boldsymbol{\sigma}_{t-\tau}$ for $\tau>0$ (Pillow et al., 2005, 2008):

$$
I_{i t}=\phi_{i}\left(\boldsymbol{s}_{t}\right)+\sum_{j} \sum_{\tau=1}^{\tau_{\max }} L_{i j, \tau} \sigma_{j(t-\tau)},
$$

where $\phi_{i}(\boldsymbol{\sigma})$ is the stimulus drive, and the filters $\boldsymbol{L}_{i j, t a u}$ capture excitatory as well as inhibitory inputs from other cells with delay $\tau$. If the time bin is short enough, the filter $\boldsymbol{L}_{i i, \tau}$ can also be used to model the influence of the refractory period on the spiking activity. The stimulus input is also learned from data. Usually, it is a linear spatial-temporal filtering of the stimulus (Pillow et al., 2008): $\phi_{i}\left(\boldsymbol{s}_{t}\right)=\boldsymbol{k}_{i}^{\top} \boldsymbol{s}_{t}$, where $k_{i}$ is a filter and $\boldsymbol{s}_{t}$ is a vector containing all the features of the stimulus at time $t$ as well as its recent past up to $t-\tau+1$. Quadratic functions of 
the stimulus have also shown to model accurately cat V1 cells (Park et al., 2013a).

The generalized linear model (GLM) is one of the most popular causal models, and is similar to the Linear Nonlinear Poisson model of neural response to stimuli (Simoncelli et al., 2004). In the GLM, the response of the cell is a stochastic process with a firing rate $\lambda_{i}$. This rate only depends on the current value of the input current $I$ :

$$
\lambda_{i t}=f_{i}\left(I_{i t}\right)
$$

where $f_{i}$ is the rate function. Typically, the rate function is an exponential, as this choice drastically simplifies computations:

$$
f_{i}(I)=\exp \left(\mu_{i}+I\right)
$$

where $\mu_{i}$ is a parameter controlling the firing rate in the absence of input. $f_{i}$ can also be chosen to be a sigmoid function to account for saturation, or a function with a sub-exponential growth to avoid unrealistically high firing rates. Responses $\sigma_{i t}$ are generated by a stochastic noise process with mean $\lambda_{i t}$ (similarly to previously discussed models): Bernouilli for binary variables and Poisson or more general models of variability for spike counts (Gao et al., 2015).

Note that the firing rate at time $t$ depends on filtered population responses at time $t-1$. Thus, contrary to previous models with interactions between neurons in a same time bin, here there is no interaction between neurons in the same time bin. As a consequence, a GLM is simpler to learn than an Ising model, as it can be learned for each neuron independently.

The GLM is usually learned by maximizing the likelihood of observed responses. If the rate function $f_{i}$ is $\log$ concave, this problem is convex. For small time bins where the firing rate is low, learning a GLM with a Bernoulli or Poisson process is equivalent (Truccolo et al., 2005). A number of priors can be added to the problem to constrain interactions to be sparse or filters to be smooth (Stevenson et al., 2009). Using such priors, Stevenson et al. (2009) showed that groups of neurons in macaque primary motor and premotor cortices had similar inputs and impact on other neurons. These groups were interpreted as functional assemblies.

As already discussed in the context of single-bin models of activity, It is possible to infer a time-dependent model instead of a stimulus-dependent model when a model to map stimulus to responses is not known, by replacing $\phi_{i}\left(\boldsymbol{s}_{t}\right)$ by a more general $\phi_{i t}$ learned from data (Roudi and Hertz (2011), where the model is called kinetic Ising with time-dependent fields). Cui et al. (2016) showed that the GLM can be further improved by adding an input corresponding to a filter applied to the LFP. This is of particular interest, as Kavser et al. (2009) showed that taking the LFP phase into account drastically improved decoding from spike patterns.
GLMs can make precise predictions for responses in a single time bin, given the population response in previous time bins. This performance has been shown in multiple neural systems, such as macaque and human primary motor cortex (Truccolo et al., 2005), macaque retina (Pillow et al., 2008) and macaque middle temporal area (Cui et al., 2016).

Although GLMs can predict single time bins, in general they cannot be used for generating data, as they tend to be very unstable (Gerhard et al., 2017; Hocker and Park, 2017). If one generates synthetic data by drawing the activity in each time bin conditioned on the synthetic data already generated in previous time bins, neural trajectories usually converge to fixed points with unrealistically high firing rates. Gerhard et al. (2017) proposed tools to analyze the stability of GLMs, along with inference methods to constrain GLMs to be stable. Hocker and Park (2017) showed that stability could also be achieved by maximizing the likelihood of not only single time bins given their past, but also of the following time bins.

The leaky integrate and fire model is very similar to the GLM (Bohte et al., 2000), in the sense that it shares the same input current $I$ (eq. 30). The difference lies in the spike generation process, which is closer to realistic biological mechanisms. The input current drives fluctuations in the membrane potential $V_{i}$, and a spike is emitted when the membrane potential reaches a threshold $V_{\mathrm{th}}$. The membrane potential evolves according to:

$$
V_{i t}=(1-\gamma) V_{i, t-1}+I_{i t}+\epsilon_{i t}
$$

where $\gamma$ sets how fast the membrane potential goes back to 0 in the absence of input. $\epsilon$ is a random, usually Gaussian, fluctuation called noise innovation. When $V_{i t}$ reaches $V_{\mathrm{th}}, V_{i, t+1}$ is set back to 0 . Cocco et al. (2009) proposed efficient techniques to infer parameters by likelihood maximization. Using a simple parametrization of the spike-history filters, $L_{i j, \tau}=w_{i j} L_{\tau}$, they found that the interaction weights $w_{i j}$ correlated strongly with the coupling coefficients $J_{i j}$ inferred from an Ising model. The same leaky integrate and fire model is used in the tempotron (Gütig and Sompolinsky, 2006), where it is trained to discriminate different correlations in input spike trains.

Bánvai et al. (2016) found that for cells in macaque $\mathrm{V} 1$, the leaky integrate and fire model was a more accurate description of neural temporal correlations than the GLM. Multiple variations of the spiking process have been proposed (Teeter et al., 2017), with different degrees of similitude to biological processes, although more detailed models tend to be harder to learn.

In practice, the GLM is more commonly used in the literature than the leaky integrate and fire model. Although GLMs offer a coarser description of responses, and predict poorly some response statistics such as interspike intervals (Hocker and Park, 2017), they are more convenient to use and to learn from data. 


\section{Transition models with latent dynamics}

The transition models presented above can account for some correlations between recorded neurons. Often, recorded neurons are part of a larger population, especially in the cortex. In this larger population, neural dynamics can have important effects. The effect of such dynamics can be included in more general transition models, in the form of dynamical latent variables. In practice, these models correspond to GLM rather than leaky integrate and fire neurons, as they are simpler to learn. This kind of model is sometimes called generalized linear model augmented with a statespace model (Vidne et al., 2012). Pillow and Latham (2007) presented methods to infer such GLM in the simple case of a single recorded neuron interacting with a single latent neuron. Using simulations, they showed that taking into account the latent neuron improved model predictions. Augmented GLM have also proven very successful at decoding and predicting correlations between neurons in the retina (Vidne et al., 2012) in V1 (Archer et al., 2014) or in motor cortex (Lawhern et al., 2010; Macke et al., 2011).

There are several ways to account for latent dynamics in the GLM input current (eq. 30). A simple way is to take a latent variable $\boldsymbol{z}$ following a Gaussian autoregressive process (eq. 27), and add the influence of the latent variable to the input current $I$ (Kulkarni and Paninski, 2007; Lawhern et al., 2010).

$$
I_{i t}=\phi_{i}\left(\boldsymbol{s}_{t}\right)+\sum_{j} \sum_{\tau=1}^{\tau_{\max }} L_{i j, \tau} \sigma_{j(t-\tau)}+\boldsymbol{C}_{i}^{\boldsymbol{\top}} \boldsymbol{z}_{t}
$$

where $\boldsymbol{C}_{i}$ is a projection vector, measuring how the latent variable influences neuron $i$. This model is a combination of the GLM (eq. 30) with a linear dynamical system (eq. 29). This model form is not easy to infer, but algorithms using expectation-maximization techniques have been proposed (Dempster et al., 1977; Kulkarni and Paninski, 2007).

If the neural population is large, or if neurons are from different cortical areas, it can be interesting to use a latent space of high dimension. However, large latent spaces make the inference hard. To simplify the inference of latent dynamics, Vidne et al. (2012) constrained the latent dynamics to be composed of multiple independent dynamics of lower dimensions. In practice, this can be achieved by separating latent dimensions in batches, and constraining the transition coefficient $A_{n m}$ (governing the dynamics of latent variable, eq. 27) to be 0 if $n$ and $m$ belong to different batches. Vidne et al. (2012) showed that for a population of 25 parasol cells in the macaque retina, the latent variable was more important than the population spiking history current $I_{\text {pop }}$ to reproduce neural correlations.

The stimulus is also likely to influence the dynamics of neighboring, unrecorded neurons. In some models, the stimulus also influences the latent variable dynamics:

$$
\boldsymbol{z}_{t}=\boldsymbol{A} \boldsymbol{z}_{t-1}+\boldsymbol{\varphi}\left(\boldsymbol{s}_{t}\right)+\boldsymbol{\epsilon}_{t}
$$

Here $\varphi$ is a function of the stimulus, with same dimensionality as the latent variable. For simplicity, the function $\varphi$ is often chosen to be linear (Archer et al., 2014; Park et al., 2015), although more complex functions have been proposed, such as quadratic and with multiplicative interactions (Archer et al., 2014). Again, in the absence of a known model to relate stimuli to responses, a timedependent variable $\varphi_{t}$ can be used instead of a stimulusdependent one (Macke et al., 2011).

In the above-mentioned form of the stimulus (eq. 34), the direct stimulus input $\phi$ can be included (Macke et al., 2011), or not (Archer et al., 2014; Park et al., 2015). Models without direct stimulus input are convenient as they allow to have fewer receptive fields than there are neurons (Archer et al., 2014). This choice can significantly decrease the number of parameters to learn, and may be relevant for large populations of neurons in the cortex, where neurons share inputs from lower areas such as the retina or the cochlea. The population can then be considered as a "computational unit" (Archer et al., 2014).

Finally, as previously described for the modulated Poisson model (eq. 23), it can be convenient to account for slow varying fluctuations that may be specific to experiments, using a latent variable $\boldsymbol{\zeta}$ constrained to have slow variations. This can be achieved by replacing $z_{t}$ by $\boldsymbol{z}_{t}+\boldsymbol{\zeta}_{t}$ in eq. 34, while preserving the same dynamics for $\boldsymbol{z}$ (Park et al., 2015). Archer et al. (2014) showed that adding these slow variations improved the description of responses in macaque $\mathrm{V} 1$ recordings.

\section{CONCLUSION AND FUTURE DIRECTIONS}

Over the last two decades, a variety of models for population responses has been proposed. Models can have very different forms, depending on analysis goals and experimental data available. They can help us characterize and interpret features of the neural code, quantify the diversity of responses, separate the influence of the stimulus from internal effects, or estimate the robustness of stimulus encoding to response noise. Population models can provide an accurate description of the state of a neural population, even for single trials where single neurons might seem unreliable. We have presented a large number and variants of population models, but this list is not exhaustive, and combinations of the aforementioned models are possible.

The size of recorded populations increases at an unprecedented pace, and the neural system of some simple organisms can now be recorded entirely with increasing spatial and temporal resolution (see Prevedel et al. 
(2014) for C. elegans, or Dupre and Yuste (2017) for the cnidarian Hydra vulgaris). Enhanced inference techniques will be required to learn model on populations of thousands of neurons. Future models will also have to adapt to future experimental constraints: the duration of recordings is not guaranteed to grow with the size of recorded populations; the sampling rate of individual neurons may be limited by the size of the recorded area when scanning techniques are used, such as in 2-photon microscopy. On the other hand, long recordings are not guaranteed to keep track of all neurons during the whole recording duration. Future models will need to account for neurons that are only observable in some parts of experiments.

Models of neural dynamics will also need to adapt to larger populations. In the GLM for example, the number of couplings grows as the square of the population size, which will be hard to infer for large populations. Fortunately, several works have suggested that the neural activity lies in a low dimensional manifold, and many dimensionality reduction techniques have been proposed to identify low-dimensional representations (see Cunningham and $\mathrm{Yu}$ (2014) for a review). Currently, GLMs model use complex dynamics for visible neurons, while other models use simple dynamics to describe latent variables of low dimensionality. Future works will need to model the dynamics of low-dimensional representations of observed neural responses. Such models of neural dynamics will need to be translated into accurate models of spike trains. The framework of latent dynamical systems could be useful for this goal, using low-dimensional representations of the population activity instead of latent variables.

Most neural models presented here are applied to populations of neurons in a single area, for animals stimulated by a single stimulus. Future model with need to account for interactions within each area along with interactions between multiple areas. A major challenge is also to account for the diversity of stimuli or even of neural states present in the recordings, and recapitulate this diversity in a single model. It is still challenging for a model to account for both awake animals and slowwave sleep, as neurons behave differently in these regimes (Nghiem et al., 2018). Coarse-grained, modular models such as the hierarchical model (Santos et al., 2010), with different types of interactions at different spatial scales, offer promising avenues for this feat. Another potential direction could be to develop models including both lowlevel recording techniques, such as electrode arrays and optical imaging, with high-level recordings, such as EEG and fMRI.

\section{REFERENCES}

Abeles, M., Bergman, H., Gat, I., Meilijson, I., Seidemann, E., Tishby, N., and Vaadia, E. (1995). Cortical activity flips among quasi-stationary states. Proceedings of the National Academy of Sciences.

Ahrens, M. B., Orger, M. B., Robson, D. N., Li, J. M., and Keller, P. J. (2013). Whole-brain functional imaging at cellular resolution using light-sheet microscopy. Nature methods, 10(5), 413-420.

Amari, S.-i., Nakahara, H., Wu, S., and Sakai, Y. (2003). Synchronous Firing and Higher-Order Interactions in Neuron Pool. Neural Computation, 15(1), 127-142.

Archer, E. W., Köster, U., Pillow, J. W., and Macke, J. H. (2014). Low-dimensional models of neural population activity in sensory cortical circuits. Advances in Neural Information Processing Systems 27, 27, 343-351.

Arieli, A., Sterkin, A., Grinvald, A., and Aertsen, A. (1996). Dynamics of ongoing activity: Explanation of the large variability in evoked cortical responses. Science, 273(5283), 1868-1871.

Bányai, M., Koman, Z., and Orbán, G. (2016). Response statistics dissect the contributions of different sources of variability to population activity in $\mathrm{V} 1$.

Baudot, P., Levy, M., Marre, O., Monier, C., Pananceau, M., and Frégnac, Y. (2013). Animation of natural scene by virtual eye-movements evokes high precision and low noise in V1 neurons. Frontiers in neural circuits, $\mathbf{7}$ (December), 206.

Berry, M. J., Warland, D. K., and Meister, M. (1997). The structure and precision of retinal spike trains. Proceedings of the National Academy of Sciences, 94, 5411-5416.

Bialek, W. and Ranganathan, R. (2007). Rediscovering the power of pairwise interactions. arXiv Prepr., (q-bio.QM), $1-8$.

Bohte, S. M., Spekreijse, H., and Roelfsema, P. R. (2000). The Effects of Pair-wise and Higher-order Correlations on the Firing Rate of a Postsynaptic Neuron. Neural Computation, 12(1), 153-179.

Brette, R. (2009). Generation of Correlated Spike Trains. Neural Computation, 21(1), 188-215.

Buonomano, D. V. and Maass, W. (2009). State-dependent computations: spatiotemporal processing in cortical networks. Nature Reviews Neuroscience, 10(2), 113-125.

Cafaro, J. and Rieke, F. (2010). Noise correlations improve response fidelity and stimulus encoding. Nature, 468(7326), 964-967.

Carandini, M. (2004). Amplification of trial-to-trial response variability by neurons in visual cortex. PLoS Biology, 2(9), 1483-1493.

Cocco, S., Leibler, S., and Monasson, R. (2009). Neuronal couplings between retinal ganglion cells inferred by efficient inverse statistical physics methods. Proc. Natl. Acad. Sci. U. S. A., 106(33), 14058-14062.

Cox, D. R. and Wermuth, N. (1999). Likelihood Factorizations for Mixed Discrete and Continuous Variables. Scandinavian Journal of Statistics, 26(2), 209-220.

Cui, Y., Liu, L. D., McFarland, J. M., Pack, C. C., and Butts, D. A. (2016). Inferring Cortical Variability from Local Field Potentials. Journal of Neuroscience, 36(14), 4121-4135.

Cunningham, J. P. and Yu, B. M. (2014). Dimensionality reduction for large-scale neural recordings. Nature Neuroscience, 17(11), 1500-1509. 
Dempster, A. P., Laird, N. M., and Rubin, D. B. (1977). Maximum likelihood from incomplete data via the em algorithm. Journal of the royal statistical society. Series B (methodological), pages 1-38.

Deweese, M. R. and Zador, A. M. (2004). Shared and private variability in the auditory cortex. Journal of neurophysiology, 92(3), 1840-55.

Dupre, C. and Yuste, R. (2017). Non-overlapping neural networks in hydra vulgaris. Current Biology, 27(8), 1085 1097.

Ecker, A. S., Berens, P., Cotton, R. J., Subramaniyan, M., Denfield, G. H., Cadwell, C. R., Smirnakis, S. M., Bethge, M., and Tolias, A. S. (2014). State dependence of noise correlations in macaque primary visual cortex. Neuron, 82(1), 235-248.

Faisal, A. A., Selen, L. P. J., and Wolpert, D. M. (2008). Noise in the nervous system. Nature Reviews Neuroscience, 9(april), 292-303.

Ferrari, U. (2016). Learning maximum entropy models from finite-size data sets: A fast data-driven algorithm allows sampling from the posterior distribution. Physical Review $E, \mathbf{9 4}(2), 023301$.

Ferrari, U., Obuchi, T., and Mora, T. (2017). Random versus maximum entropy models of neural population activity. Physical Review E, 95(4), 042321.

Ferrari, U., Deny, S., Chalk, M., Tkačik, G., Marre, O., and Mora, T. (2018). Separating intrinsic interactions from extrinsic correlations in a network of sensory neurons. arXiv:1801.01823

Fischer, A. and Igel, C. (2012). An Introduction to Restricted Boltzmann Machines. Lecture Notes in Computer Science: Progress in Pattern Recognition, Image Analysis, Computer Vision, and Applications, 7441, 14-36.

Franke, F., Fiscella, M., Sevelev, M., Roska, B., Hierlemann, A., and Azeredo da Silveira, R. (2016). Structures of Neural Correlation and How They Favor Coding. Neuron, 89(2), 409-422.

Ganmor, E., Segev, R., and Schneidman, E. (2011a). Sparse low-order interaction network underlies a highly correlated and learnable neural population code. Proceedings of the National Academy of Sciences of the United States of America, 108(23), 9679-84.

Ganmor, E., Segev, R., and Schneidman, E. (2011b). The architecture of functional interaction networks in the retina. The Journal of neuroscience, 31(8), 3044-54.

Ganmor, E., Segev, R., and Schneidman, E. (2015). A thesaurus for a neural population code. eLife, 4, 1-19.

Gao, Y., Buesing, L., Shenoy, K. V., and Cunningham, J. P. (2015). High-dimensional neural spike train analysis with generalized count linear dynamical systems. Advances in Neural Information Processing System, pages 1-9.

Gao, Y., Archer, E., Paninski, L., and Cunningham, J. P. (2016). Linear dynamical neural population models through nonlinear embeddings. (Nips), 1-9.

Gardella, C., Marre, O., and Mora, T. (2016). A tractable method for describing complex couplings between neurons and population rate. eNeuro, $\mathbf{3}$ (August), 1-13.

Gardella, C., Marre, O., and Mora, T. (2018). Blindfold learning of an accurate neural metric. Proc. Natl. Acad. Sci., 115(13), 3267-3272.

Gerhard, F., Deger, M., and Truccolo, W. (2017). On the stability and dynamics of stochastic spiking neuron models: Nonlinear Hawkes process and point process GLMs.

Gerwinn, S., Berens, P., and Bethge, M. (2009). A joint maximum-entropy model for binary neural population patterns and continuous signals. Advances in Neural Information Processing Systems 22, (1), 620-628.

Gollisch, T. and Meister, M. (2010). Eye Smarter than Scientists Believed: Neural Computations in Circuits of the Retina.

Granot-Atedgi, E., Tkačik, G., Segev, R., and Schneidman, E. (2013). Stimulus-dependent Maximum Entropy Models of Neural Population Codes. PLoS Computational Biology, $\mathbf{9}(3)$.

Grün, S., Diesmann, M., and Aertsen, A. (2002). Unitary events in multiple single-neuron spiking activity: II. Nonstationary data. Neural computation, 14(1), 81-119.

Grünwald, P. D. and Philip Dawid, A. (2004). Game theory, maximum entropy, minimum discrepancy and robust Bayesian decision theory. Annals of Statistics, 32(4), 13671433.

Gütig, R. and Sompolinsky, H. (2006). The tempotron: a neuron that learns spike timingbased decisions. Nature Neuroscience, 9(3), 420-428.

Hinton, G. E. (2002). Training products of experts by minimizing contrastive divergence. Neural computation, 14(8), $1771-1800$.

Hinton, G. E., Sejnowski, T. J., and Ackley, D. H. (1984). Boltzmann machines: Constraint satisfaction networks that learn.

Hocker, D. and Park, I. M. (2017). Multistep inference for generalized linear spiking models curbs runaway excitation. 8th Int. IEEE EMBS Conf. Neural Eng., (Ner).

Hopfield, J. J. (1982). Neural networks and physical systems with emergent collective computational abilities. Proceedings of the National Academy of Sciences, 79(8), 25542558.

Humplik, J. and Tkačik, G. (2016). Semiparametric energybased probabilistic models . page 8 .

Jaynes, E. T. (1957). Information Theory and Statistical Mechanics. II. Phys. Rev., 108(2), 171-190.

Kayser, C., Montemurro, M. A., Logothetis, N. K., and Panzeri, S. (2009). Spike-Phase Coding Boosts and Stabilizes Information Carried by Spatial and Temporal Spike Patterns. Neuron, 61(4), 597-608.

Köster, U., Sohl-Dickstein, J., Gray, C. M., and Olshausen, B. A. (2014). Modeling Higher-Order Correlations within Cortical Microcolumns. PLoS Computational Biology, 10(7), 1-12.

Kulkarni, J. E. and Paninski, L. (2007). Common-input models for multiple neural spike-train data. Network: Computation in Neural Systems, 18(4), 375-407.

Lawhern, V., Wu, W., Hatsopoulos, N., and Paninski, L. (2010). Population decoding of motor cortical activity using a generalized linear model with hidden states. Journal of Neuroscience Methods, 189(2), 267-280.

Le Roux, N. and Bengio, Y. (2008). Representational Power of Restricted Boltzmann Machines and Deep Belief Networks. Neural Computation, 20(6), 1631-1649.

Lin, I. C., Okun, M., Carandini, M., and Harris, K. D. (2015). The Nature of Shared Cortical Variability. Neuron, 87(3), 644-656.

Loback, A. R., Prentice, J. S., Ioffe, M. L., and Berry, M. J. (2018). Noise-Robust Modes of the Retinal Population Code Have the Geometry of Ridges and Correspond to Neuronal Communities. Neural Comput., 29, 3119-3180.

Longtin, A., Bulsara, A., and Moss, F. (1991). Time-interval sequences in bistable systems and the noise-induced trans- 
mission of information by sensory neurons. Physical Review Letters, 67(5), 656.

Luczak, A., Bartho, P., and Harris, K. D. (2013). Gating of sensory input by spontaneous cortical activity. Journal of Neuroscience, 33(4), 1684-1695.

Lyamzin, D. R., Barnes, S. J., Donato, R., Garcia-Lazaro, J. A., Keck, T., and Lesica, N. A. (2015). Nonlinear Transfer of Signal and Noise Correlations in Cortical Networks. Journal of Neuroscience, 35(21), 8065-8080.

Macke, J. H., Berens, P., Ecker, A. S., Tolias, A. S., and Bethge, M. (2009). Generating spike trains with specified correlation coefficients. Neural Computation, 21(2), 397423.

Macke, J. H., Buesing, L., Cunningham, J. P., Yu, B. M., Shenoy, K. V., and Sahani, M. (2011). Empirical models of spiking in neural populations. Advances in Neural Information Processing Systems, 24, 1350-1358.

Mainen, Z. F. and Sejnowski, T. J. (1995). Reliability of spike timing in neocortical neurons. Science, 268(5216), 1503-6.

Marre, O., El Boustani, S., Frégnac, Y., and Destexhe, A. (2009). Prediction of spatiotemporal patterns of neural activity from pairwise correlations. Physical Review Letters, 102(13), 5-8.

Martignon, L., Von Hassein, H., Grün, S., Aertsen, A., and Palm, G. (1995). Detecting higher-order interactions among the spiking events in a group of neurons. Biological Cybernetics, 73(1), 69-81.

Mazor, O. and Laurent, G. (2005). Transient dynamics versus fixed points in odor representations by locust antennal lobe projection neurons. Neuron, 48(4), 661-673.

McIntosh, L., Maheswaranathan, N., Nayebi, A., Ganguli, S., and Baccus, S. (2016). Deep learning models of the retinal response to natural scenes. In Advances in Neural Information Processing Systems, pages 1369-1377.

Meshulam, L., Gauthier, J. L., Brody, C. D., Tank, D. W., and Bialek, W. (2017). Collective Behavior of Place and Non-place Neurons in the Hippocampal Network. Neuron, 96(5), 1178-1191.e4.

Montani, F., Ince, R. A. A., Senatore, R., Arabzadeh, E., Diamond, M. E., and Panzeri, S. (2009). The impact of highorder interactions on the rate of synchronous discharge and information transmission in somatosensory cortex. Philosophical Transactions of the Royal Society A: Mathematical, Physical and Engineering Sciences, 367(1901), 32973310.

Montemurro, M. A., Senatore, R., and Panzeri, S. (2007). Tight Data-Robust Bounds to Mutual Information Combining Shuffling and Model Selection Techniques. Neural Computation, 19(11), 2913-2957.

Mora, T., Deny, S., and Marre, O. (2015). Dynamical criticality in the collective activity of a population of retinal neurons. Physical Review Letters, 114(7).

Nasser, H., Marre, O., and Cessac, B. (2013). Spatio-temporal spike train analysis for large scale networks using the maximum entropy principle and Monte Carlo method. Journal of Statistical Mechanics: Theory and Experiment, pages $1-41$.

Nemenman, I., Lewen, G. D., Bialek, W., and De Ruyter Van Steveninck, R. R. (2008). Neural coding of natural stimuli: Information at sub-millisecond resolution. PLoS Computational Biology, 4(3).

Nghiem, T.-a., Tele, B., Marre, O., Destexhe, A., and Ferrari, U. (2018). Maximum entropy models reveal the correlation structure in cortical neural activity during wakefulness and sleep. arXiv Prepr., pages 1-16.

Nienborg, H., R. Cohen, M., and Cumming, B. G. (2012). Decision-related activity in sensory neurons: correlations among neurons and with behavior. Annual review of neuroscience, 35, 463-483.

Nowak, L. G., Sanchez-Vives, M. V., and McCormick, D. A. (1997). Influence of low and high frequency inputs on spike timing in visual cortical neurons. Cerebral cortex (New York, NY: 1991), 7(6), 487-501.

Ohiorhenuan, I. E., Mechler, F., Purpura, K. P., Schmid, A. M., Hu, Q., and Victor, J. D. (2010). Sparse coding and high-order correlations in fine-scale cortical networks. Nature, 466(7306), 617-621.

Okun, M., Steinmetz, N. a., Cossell, L., Iacaruso, M. F., Ko, H., Barthó, P., Moore, T., Hofer, S. B., Mrsic-Flogel, T. D., Carandini, M., and Harris, K. D. (2015). Diverse coupling of neurons to populations in sensory cortex. Nature, 521(7553), 511-515.

ODonnell, C., Gonalves, J. T., Whiteley, N., Portera-Cailliau, C., and Sejnowski, T. J. (2017). The population tracking model: A simple, scalable statistical model for neural population data. Neural Computation, 29(1), 50-93.

Panzeri, S., Petersen, R. S., Schultz, S. R., Lebedev, M., and Diamond, M. E. (2001). The Role of Spike Timing in the Coding of Stimulus Location in Rat Somatosensory Cortex. In Neuron, volume 29, pages 769-777.

Park, I. M., Archer, E. W., Priebe, N., and Pillow, J. W. (2013a). Spectral methods for neural characterization using generalized quadratic models. Advances in Neural Information Processing Systems, 26, 2454-2462.

Park, I. M., Archer, E. W., Latimer, K., and Pillow, J. W. (2013b). Universal models for binary spike patterns using centered Dirichlet processes. Advances in Neural Information Processing Systems 26, 26, 2463-2471.

Park, M., Bohner, G., and Macke, J. H. (2015). Unlocking neural population non-stationarities using hierarchical dynamics models. Advances in Neural Information Processing Systems, pages 145-153.

Pillow, J. W. and Latham, P. (2007). Neural characterization in partially observed populations of spiking neurons. Neural Information Processing Systems, pages 1-9.

Pillow, J. W., Paninski, L., Uzzell, V. J., Simoncelli, E. P., and Chichilnisky, E. (2005). Prediction and decoding of retinal ganglion cell responses with a probabilistic spiking model. Journal of Neuroscience, 25(47), 11003-11013.

Pillow, J. W., Shlens, J., Paninski, L., Sher, A., Litke, A. M., Chichilnisky, E. J., and Simoncelli, E. P. (2008). Spatiotemporal correlations and visual signalling in a complete neuronal population. Nature, 454(7207), 995-999.

Prentice, J. S., Marre, O., Ioffe, M. L., Loback, A. R., Tkačik, G., and Berry II, M. J. (2016). Error-Robust Modes of the Retinal Population Code. PLOS Computational Biology, 12(11), e1005148.

Prevedel, R., Yoon, Y.-G., Hoffmann, M., Pak, N., Wetzstein, G., Kato, S., Schrödel, T., Raskar, R., Zimmer, M., Boyden, E. S., and Vaziri, A. (2014). Simultaneous wholeanimal 3D imaging of neuronal activity using light-field microscopy. Nature Methods, 11(7), 727-730.

Puchalla, J. L., Schneidman, E., Harris, R. a., and Berry, M. J. (2005). Redundancy in the population code of the retina. Neuron, 46(3), 493-504.

Rabinowitz, N. C., Goris, R. L. T., Ballé, J., and Simoncelli, E. P. (2015). A model of sensory neural responses in the presence of unknown modulatory inputs. 2014, 1-9. 
Reich, D. S., Victor, J. D., Knight, B. W., Ozaki, T., and Kaplan, E. (1997). Response variability and timing precision of neuronal spike trains in vivo. Journal of neurophysiology, 77(5), 2836-2841.

Reinagel, P. and Reid, R. C. (2000). Temporal coding of visual information in the thalamus. Journal of Neuroscience, 20(14), 5392-5400.

Reyes, A. D. (2003). Synchrony-dependent propagation of firing rate in iteratively constructed networks in vitro. Nature neuroscience, 6(6), 593-9.

Roudi, Y. and Hertz, J. (2011). Mean field theory for nonequilibrium network reconstruction. Physical Review Letters, 106(4), 1-4.

Santos, G. S., Gireesh, E. D., Plenz, D., and Nakahara, H. (2010). Hierarchical Interaction Structure of Neural Activities in Cortical Slice Cultures. Journal of Neuroscience, 30(26), 8720-8733.

Schaub, M. T. and Schultz, S. R. (2012). The Ising decoder: reading out the activity of large neural ensembles. Journal of computational neuroscience, 32(1), 101-18.

Schneidman, E., Still, S., Berry, M. J., and Bialek, W. (2003). Network information and connected correlations. Phys. Rev. Lett., 91(23), 238701.

Schneidman, E., Berry, M. J., Segev, R., and Bialek, W. (2006). Weak pairwise correlations imply strongly correlated network states in a neural population. Nature, 440(7087), 1007-1012.

Schneidman, E., Puchalla, J. L., Segev, R., Harris, R. a., Bialek, W., and Berry II, M. J. (2011). Synergy from silence in a combinatorial neural code. Journal of neuroscience, 31(44), 15732-41.

Schölvinck, M. L., Saleem, A. B., Benucci, A., Harris, K. D., and Carandini, M. (2015). Cortical state determines global variability and correlations in visual cortex. Journal of Neuroscience, 35(1), 170-178.

Schwartz, G., Macke, J., Amodei, D., Tang, H., and Berry, M. J. (2012). Low error discrimination using a correlated population code. Journal of neurophysiology, 108(4), 1069-88.

Shepherd, G. (2004). The Synaptic Organization of the Brain. Oxford University Press, USA.

Shlens, J., Field, G. D., Gauthier, J. L., Grivich, M. I., Petrusca, D., Sher, A., Litke, A. M., and Chichilnisky, E. J. (2006). The Structure of Multi-Neuron Firing Patterns in Primate Retina. Journal of Neuroscience, 26(32), 82548266.

Shlens, J., Field, G. D., Gauthier, J. L., Greschner, M., Sher, A., Litke, A. M., and Chichilnisky, E. J. (2009). The Structure of Large-Scale Synchronized Firing in Primate Retina. Journal of Neuroscience, 29(15), 5022-5031.

Shu, Y., Hasenstaub, A., Badoual, M., Bal, T., and McCormick, D. A. (2003). Barrages of synaptic activity control the gain and sensitivity of cortical neurons. Journal of Neuroscience, 23(32), 10388-10401.

Simoncelli, E. P., Paninski, L., Pillow, J., and Schwartz, O. (2004). Characterization of neural responses with stochastic stimuli. The cognitive neurosciences, 3, 327-338.

Singer, W. and Gray, C. M. (1995). Visual feature integration and the temporal correlation hypothesis. Annual review of neuroscience, 18(1), 555-86.

Smolensky, P. (1986). Parallel distributed processing: Explorations in the microstructure of cognition, vol. 1: Foundations. chapter Chapter 6: Information Processing in Dynamical Systems: Foundations of Harmony Theory. MIT
Press, Cambridge, MA, USA.

Socolich, M., Lockless, S. W., Russ, W. P., Lee, H., Gardner, K. H., and Ranganathan, R. (2005). Evolutionary information for specifying a protein fold. Nature, 437(7058), $512-518$.

Stevenson, I. H., Rebesco, J. M., Hatsopoulos, N. G., Haga, Z., Miller, L. E., and Körding, K. P. (2009). Bayesian inference of functional connectivity and network structure from spikes. IEEE Trans. Neural Systems and Rehab., 17(3), $1-10$.

Stringer, C., Pachitariu, M., Steinmetz, N. A., Okun, M., Bartho, P., Harris, K. D., Sahani, M., and Lesica, N. A. (2016). Inhibitory control of correlated intrinsic variability in cortical networks. eLife, 5(DECEMBER2016), 1-33.

Tang, A., Jackson, D., Hobbs, J., Chen, W., Smith, J. L., Patel, H., Prieto, A., Petrusca, D., Grivich, M. I., Sher, A., Hottowy, P., Dabrowski, W., Litke, A. M., and Beggs, J. M. (2008). A Maximum Entropy Model Applied to Spatial and Temporal Correlations from Cortical Networks In Vitro. Journal of Neuroscience, 28(2), 505-518.

Teeter, C., Iyer, R., Menon, V., Gouwens, N., Feng, D., Berg, J., Cain, N., Koch, C., and Mihalas, S. (2017). Generalized Leaky Integrate-And-Fire Models Classify Multiple Neuron Types. bioRxiv.

Tieleman, T. (2008). Training Restricted Boltzmann Machines using Approximations to the Likelihood Gradient. Proceedings of the 25th International Conference on $\mathrm{Ma}$ chine Learning, 307, 7.

Tkačik, G., Prentice, J. S., Balasubramanian, V., and Schneidman, E. (2010). Optimal population coding by noisy spiking neurons. Proceedings of the National Academy of Sciences of the United States of America, 107, 14419-14424.

Tkačik, G., Marre, O., Mora, T., Amodei, D., Berry II, M. J., and Bialek, W. (2013). The simplest maximum entropy model for collective behavior in a neural network. Journal of Statistical Mechanics: Theory and Experiment, 2013(03), P03011.

Tkacik, G., Marre, O., Amodei, D., Schneidman, E., Bialek, W., and Berry II, M. J. (2014). Searching for Collective Behavior in a Large Network of Sensory Neurons. PLoS Comput Biol, 10(1), e1003408.

Tkacik, G., Mora, T., Marre, O., Amodei, D., Palmer, S. E., Berry II, M. J., and Bialek, W. (2015). Thermodynamics and signatures of criticality in a network of neurons. 112(37), 11508-11513.

Truccolo, W., Eden, U. T., Fellows, M. R., Donoghue, J. P., and Brown, E. N. (2005). A Point Process Framework for Relating Neural Spiking Activity to Spiking History, Neural Ensemble, and Extrinsic Covariate Effects. Journal of Neurophysiology, 93(2), 1074-1089.

Truccolo, W., Hochberg, L. R., and Donoghue, J. P. (2010). Collective dynamics in human and monkey sensorimotor cortex: predicting single neuron spikes. Nature Neuroscience, 13(1), 105-111.

van Vreeswijk, C. and Sompolinsky, H. (1996). Chaos in neuronal networks with balanced excitatory and inhibitory activity. Science, 274(5293), 1724-1726.

Vasquez, J. C., Marre, O., Palacios, A. G., Berry, M. J., and Cessac, B. (2012). Gibbs distribution analysis of temporal correlations structure in retina ganglion cells. Journal of Physiology Paris, 106(3-4), 120-127.

Vidne, M., Ahmadian, Y., Shlens, J., Pillow, J. W., Kulkarni, J., Litke, A. M., Chichilnisky, E. J., Simoncelli, E., and Paninski, L. (2012). Modeling the impact of com- 
mon noise inputs on the network activity of retinal ganglion cells. Journal of Computational Neuroscience, 33(1), 97-121.

Vogels, T. P., Rajan, K., and Abbott, L. F. (2005). Neural Network Dynamics. Annual Review of Neuroscience, 28(1), 357-376.

Warland, D. K., Reinagel, P., and Meister, M. (1997). Decoding visual information from a population of retinal ganglion cells. Journal of neurophysiology, 78(5), 2336-2350.

Watanabe, T., Hirose, S., Wada, H., Imai, Y., Machida, T., Shirouzu, I., Konishi, S., Miyashita, Y., and Masuda, N. (2014). Energy landscapes of resting-state brain networks. Front. Neuroinform., 8(February), 12.

Wehr, M. and Laurent, G. (1996). Odour encoding by temporal sequences of firing in oscillating neural assemblies. Nature, 384(6605), 162-166.

Wehr, M. and Zador, A. M. (2003). Balanced inhibition underlies tuning and sharpens spike timing in auditory cortex.
Nature, 426(6965), 442.

Wolf, S., Supatto, W., Debrégeas, G., Mahou, P., Kruglik, S. G., Sintes, J.-M., Beaurepaire, E., and Candelier, R. (2015). Whole-brain functional imaging with two-photon light-sheet microscopy. Nature methods, 12(5), 379-380.

Yu, B., Cunningham, J., Santhanam, G., Ryu, S., Shenoy, K., and Sahani, M. (2009). Gaussian-Process Factor Analysis for Low-Dimensional Single-Trial Analysis of Neural Population Activity. Journal of Neurophysiology, 102(April 2009), 614-635.

Yu, S., Yang, H., Nakahara, H., Santos, G. S., Nikolic, D., and Plenz, D. (2011). Higher-Order Interactions Characterized in Cortical Activity. Journal of Neuroscience, 31(48), 17514-17526.

Zylberberg, J., Cafaro, J., Turner, M. H., Shea-Brown, E., and Rieke, F. (2016). Direction-selective circuits shape noise to ensure a precise population code. Neuron, $\mathbf{8 9}(2)$, 369-383. 